OPEN ACCESS

Citation: Dario Tuorto (2021) II disallineamento tra orientamenti elettorali e posizioni sui temi: una questione giovanile o di famiglia politica? Quaderni dell'Osservatorio elettorale - Italian Journal of Electoral Studies 84(2): 9-23. doi: 10.36253/qoe-10755

Received: April 8, 2021

Accepted: August 10, 2021

Published: September 6, 2021

Copyright: (c) 2021 Dario Tuorto. This is an open access, peer-reviewed article published by Firenze University Press (http://www.fupress.com/qoe) and distributed under the terms of the Creative Commons Attribution License, which permits unrestricted use, distribution, and reproduction in any medium, provided the original author and source are credited.

Data Availability Statement: All relevant data are within the paper and its Supporting Information files.

Competing Interests: The Author(s) declare(s) no conflict of interest.

Orcid

DT: 0000-0002-3655-0528

\section{Il disallineamento tra orientamenti elettorali e posizioni sui temi: una questione giovanile o di famiglia politica?}

\author{
DARIO TUORTO \\ University of Bologna, Italy \\ Email: dario.tuorto@unibo.it
}

\begin{abstract}
The transformation of politics in contemporary democracies has led to the emergence of a new ideological conflict, alongside the traditional left-right scheme, described as liberal-authoritarian or cosmopolitan-nationalist cleavage (Kriesi et al. 2008; Hooghe and Marks 2002). This change brought to a redefinition of the linkages between issue and voting preferences, as many voters decide to support a party regardless of their positions on issues while voting for the same party. Within such framework, the contribute of the new generations to the growth of the electoral dealignment and volatility has been largely analysed (Miller et al. 1996; Franklin 2004; Plutzer 2002). Issue incongruency is part of the process. Young people are often considered to be tolerant and inclusive because they grew up under prosperous and secure conditions and developed post-materialist values of freedom, multiculturalism, progressivism (Inglehart and Welzel 2005; Janmaat and Keating 2019). However, the perspective of left-cosmopolitans engaged in electoral politics contrasts with the image of economically-insecure left-behind group of young people who don't share the same progressive values (Bartle et al. 2020; Sloam and Henn 2019) and support right-wing political parties. What is still unknown is the extent to which extreme ideological traits and attitudes (e.g. negative discourses on immigration) combine with positions of openness on individual freedom. Likewise, the same contradiction can be found among left-wing voters who assume liberal position on economy or those economically left and culturally conservative. The article aims at analysing the relationship between issue positions and vote (propensity to vote). We test the hypotheses of a coherent vs incoherent ideological space by looking at the structure of voters' preferences on economic (State vs. free market) as well as cultural issues (individual rights, attitudes towards minorities, European integration) and the differences between young people and older component of the electorate. The analysis is focused on the Italian case. Data are taken from the 2020 Itanes survey.
\end{abstract}

Keywords: issue preferences, economic issues, cultural issues, young voters.

\section{IL DIBATTITO SULLA TRASFORMAZIONE DELLO SPAZIO POLITICO}

La letteratura sulla scelta di voto si è a lungo soffermata a studiare le preferenze degli elettori sui temi politici più controversi. L’assunto alla base 
dei diversi approcci che prendono in considerazione le issues, in particolare i modelli spaziali (Downs 1957; Rabinowitz e MacDonald 1989), è che esista una congruenza tra posizione individuale e posizione attribuita al partito votato. A partire da questo confronto, e dal riconoscimento della congruenza, gli elettori decidono di votare la formazione che rende possibile un voto "corretto", ossia corrispondente ai propri valori e alle proprie priorità (Lau e Redlawsk 1997; Lau et al. 2014). Secondo tale schema, i partiti propongono programmi politici chiaramente confrontabili tra loro, gli elettori scelgono in base alla vicinanza programmatica con i partiti e, se il voto avviene coerentemente con le posizioni espresse sui temi, si realizza una coincidenza tra preferenze individuali e piattaforme dei partiti (Kurella e Rosset 2018). Laspetto problematico di questo meccanismo apparentemente logico e funzionale è che non risulta sempre possibile per l'elettore stabilire la connessione ipotizzata. In primo luogo, le caratteristiche dei partiti e del sistema politico possono produrre incongruenze molteplici che rendono meno facile il riconoscimento reciproco delle posizioni. Da ciò deriva, ad esempio, l'elaborazione di strategie di reframing funzionali a spostare l'attenzione da specifiche issue o a selezionarne solo alcune (Lefevere et al. 2019; Walgrave et al. 2014). L'incongruenza può derivare, poi, dal fatto che gli elettori dispongono di informazioni imperfette, che non consentono loro di accedere a tutti gli strumenti utili a conoscere le posizioni dei partiti per arrivare poi ad elaborare una scelta corretta (o più corretta di altre). Esiste, però, una questione più generale in grado di interferire sul processo di stabilizzazione della decisione di voto. Il riferimento è alla trasformazione dello spazio politico, delle dimensioni che lo costituiscono e, quindi, del modo in cui partiti ed elettori si vanno a collocare in questo spazio.

Per lungo tempo la distinzione sinistra-destra ha funzionato come unica o principale categoria utilizzata per la competizione elettorale (Fuchs e Klingemann 1990; Klingemann, et al. 1994; Knutsen 1995). All'interno di uno spazio politico unidimensionale le posizioni dei partiti sulle molteplici e potenziali linee di conflitto coincidevano sostanzialmente con le posizioni assunte sull'asse economico, quello che opponeva Stato e mercato, pubblico e privato. Gli elettori traducevano facilmente le preferenze in scelte di voto perché lo spazio era relativamente semplice da interpretare e da occupare (Pierce 1999; Louwerse e Andeweg 2020). Il polo progressista accoppiava aspirazione all'eguaglianza e pluralismo culturale (posizione socialist-libertarian), mentre il polo opposto enfatizzava l'economia di mercato e l'uniformità culturale (capitalist-autoritharian) (Hooghe et al. 2002). Da parte loro, gli elettori sceglievano il partito da vota- re grazie alla presenza della bussola ideologica sinistradestra, attraverso cui era possibile prevedere la posizione su tutti principali temi politici.

Il declino dei cleavages tradizionali (Dalton 1984; Franklin 1992; Franklin et al. 2009), intensificatosi a partire dagli anni novanta, ha determinato la rottura di questa relazione stringente soprattutto per quanto riguarda il legame tra classe sociale e voto. Pur non potendo ancora decretare l'irreversibilità del cambiamento in atto, è indubbio che lo spazio di competizione politica sia diventato via via più complesso, strutturato in dimensioni non sempre sovrapponibili, rispetto a cui gli elettori si posizionano in modo non definitivo. In particolare, numerosi autori hanno posto l'attenzione sull'emergere di una nuova divisione centrata sui temi culturali (Bornschier 2010; Kitschelt 2004), diversa da quella rappresentata in passato lungo l'asse secolarereligioso in quanto largamente disancorata da una base sociale di riferimento e dal posizionamento sinistradestra. Per Hooghe e colleghi (2002), una polarità di questa nuova linea di divisione è stata occupata dalle formazioni della destra radicale (i partiti TAN, traditional-authoritarian-nationalist) che sponsorizzano posizioni di chiusura, mentre all'estremo opposto si collocano i partiti GAL (Green-alternative-libertarian) a favore dell'apertura. La contrapposizione si manifesta, secondo Bornschier e Kriesi, tra valori libertari e transnazionali da un lato, valori di difesa del nazionalismo e particolarismo dall'altro (Bornschier e Kriesi 2012).

Come evidenziato da questi autori, se negli anni settanta e ottanta il cambiamento politico era stato promosso dalla new left con l'irruzione nell'agenda politica dei temi ecologisti, sulla libertà di scelta in campo sessuale e sui diritti civili, negli anni più recenti è stata la destra a completare la disarticolazione dello spazio politico, per certi versi in reazione a questi cambiamenti. Mentre il conflitto distributivo sull'economia ha perso progressivamente rilevanza, una nuova polarizzazione è emersa attorno ai temi che hanno a che fare con i valori e con il concetto di comunità. Lo spazio politico bidimensionale alterato che si è andato a formare ha cambiato il significato della contrapposizione politico-ideologica (Kriesi et al. 2006; 2008). Sul piano economico, la divisione Stato-mercato ha incorporato la dimensione del protezionismo dal lato dello Stato e della competizione globale dal lato del mercato. Sul piano culturale, invece, i temi dell'immigrazione e dell'integrazione europea hanno accresciuto la loro salienza facendo lievitare i consensi e la reputazione dei partiti che li hanno cavalcati. Secondo diversi autori (Van der Brug e Van Spanje 2009; Hooghe e Marks 2002; 2018) è la contrapposizione tra i gruppi dei winners - élite, nuova classe media 
di professionals e di creativi avvantaggiati dall'apertura dei mercati - e dei losers - operai, precari, componenti di middle class tradizionale spiazzati dalle trasformazioni economiche - ad avere reso la dimensione culturale decisiva nello scenario politico contemporaneo dei paesi occidentali.

Dal lato dei partiti, il cambiamento ha investito in primo luogo le formazioni mainstream. Socialisti e conservatori si sono orientati favorevolmente rispetto al mercato e alla globalizzazione, distinguendosi più sul piano culturale che su quello economico e rinunciando quindi a interpretare (e a innovare) questa dimensione del conflitto. L'effetto è stato il rafforzamento dei partiti challenging in tutte le direzioni politiche, dai Verdi alla populist radical right a nuove formazioni non connotate sul piano ideologico ma in grado di spostare l'attenzione su questioni non presidiate da altri attori.

Dentro questo scenario sono soprattutto i partiti populisti e della destra radicale ad avere conquistato spazio, nonostante la loro base elettorale sia divisa tra piccola borghesia che sostiene politiche liberiste e working class, spesso di provenienza socialista, più favorevole a politiche stataliste. Ed è proprio la compresenza di bacini di consenso alternativi a spiegare la progressiva sostituzione dei toni pro-mercato a favore di posizioni nazionaliste (Kitschelt 2004; Van der Brug 2009; Kurella e Rosset 2018). Per quanto riguarda i temi culturali, invece, le formazioni di quest'area politico-ideologica hanno espresso posizioni disomogenee, in alcuni casi di (parziale) apertura su singoli diritti e libertà individuali - ad esempio di difesa della democrazia liberale in chiave anti-islamica (Mudde 2007) - o che coniugano autoritarismo e richiesta di partecipazione diretta dei cittadini (De Lange 2007). Guardando al campo politico opposto, alcuni autori hanno evidenziato il potenziale di posizioni come quella del left-authoritarianism (statalismo sulle policies socio-economiche, conservatorismo sui valori), ancora poco coperte dai partiti di sinistra e potenzialmente strategiche per recuperare voti tra l'elettorato operaio e a basso titolo di studio (Van der Brug e Van Spanje 2009). Una contraddizione speculare si riscontra tra i partiti left-libertarian nella misura in cui sostengono il superamento del modello tradizionale di welfare, la parziale liberalizzazione del mercato del lavoro e la redistribuzione dei benefici a vantaggio delle nuove generazioni (Kitschelt 2004).

Il passaggio a una struttura più complessa dello spazio politico ha fatto sì che le preferenze sui temi culturali non dipendano più automaticamente dalla posizione sui temi economici (Kriesi et al. 2006). Gli elettori si sono trovati in una situazione di pressioni incrociate, con diversi partiti che rappresentano le loro preferenze su più dimensioni e, quindi, non si adattano pienamente alle loro aspettative. Al tempo stesso, è cresciuta negli anni della crisi economica la salienza di temi come l'immigrazione e l'euroscetticismo, che hanno rafforzato le formazioni capaci di enfatizzarli strategicamente anche come single issue e allargare il consenso a elettori non connotati sul piano ideologico (Van der Brug e Fennema 2003; Werts et al. 2013; Dennison e Geddes 2019).

\section{GIOVANI E DISARTICOLAZIONE DELLE POSIZIONI SUI TEMI COME MOTORE DEL CAMBIAMENTO POLITICO}

In un quadro politico-elettorale in trasformazione grande importanza gioca il posizionamento dei giovani, non solo rispetto ai loro profili demografici ma anche rispetto agli atteggiamenti che esprimono, potenzialmente differenti da quelli delle altre classi di età. La frammentazione di posizioni che viene solitamente attribuita ai giovani deriva dal fatto che sono meno esposti a visioni ideologiche omogenee e compatte e, quindi, risultano più permeabili a messaggi provenienti da singoli temi e proposti da singoli partiti che ne fanno la loro bandiera. Il processo di de-ideologizzazione assume una dimensione generazionale nel senso che coinvolge soprattutto i giovani in quanto gruppo di elettori con meno esperienze e meno storia politica alle spalle, caratterizzati da status sociali più incerti e tendenzialmente più esposti alle influenze esterne (Plutzer 2002); quindi, con un'identità politica più debole (García-Albacete 2014). Se è vero che il voto è un'abitudine che si forma nel tempo (Franklin 2004) per poi stabilizzarsi sulla base di quello che si è costruito all'inizio della carriera elettorale, entrare sulla scena in una fase di grandi cambiamenti non può che influenzare l'identità politica nel senso dell'instabilità (Franklin e Wessels 2002), come avevano mostrato studi precedenti (Miller et al. 1996; Rubenson et al. 2004) e le note tesi sul cambiamento valoriale (Inglehart 1990; Welzel et al. 2003) e sulla crescita dei cittadini apartisan (Dalton 1984).

Nel caso italiano, queste riflessioni hanno assunto una particolare rilevanza a seguito dei bruschi cambiamenti dell'assetto partitico e delle maggioranze di governo, avvenuti a partire dal 2013, che hanno reso possibile l'espressione di preferenze di voto nuove per quegli elettori in uscita dai due poli e indisponibili a votare direttamente le formazioni del polo opposto (Schadee et al. 2019), ma anche per molti neo-elettori privi di riferimenti ideologici e di esperienze di socializzazione politica da cui attingere. 
Alcuni studi sul voto dei giovani (Maggini 2016; Tuorto 2018) hanno mostrato che sono soprattutto le nuove generazioni a disaccoppiare le issue tra loro dando vita a combinazioni nuove. L'esposizione massiccia alle informazioni provenienti dai nuovi ambienti mediatici e il processo di emancipazione femminile hanno accresciuto l'importanza di questioni che si collocano lungo la linea di divisione materialismo-post-materialismo (ambiente, diritti delle minoranze, libertà di scelta nei comportamenti sessuali, ecc.), in grado di trasformare i significati stessi assunti dall'asse sinistra-destra. Questa opportunità di espansione dello spazio di voto GAL si è concretizzata con la presenza di opzioni politiche, come quella del voto al Movimento 5 stelle, in grado di accogliere flussi di elettori di diversa provenienza.

Altro tratto emergente - e per certi versi confliggente con gli orientamenti sin qui evidenziati - è la crescita della quota di elettori giovani che si collocano al centro anche se non votano partiti centristi tradizionali come i conservatori. Questo posizionamento riflette il rifiuto e l'estraneità dall'asse sinistra-destra e investe, ad esempio, il voto ambientalista in diversi paesi (Van Haute 2016), assumendo quindi una connotazione post-ideologica e riflettendo il processo più generale di disarticolazione delle posizioni politiche. Un discorso analogo vale per il M5S, il partito su cui si sono concentrate le preferenze giovanili nel 2013, che ha raccolto sin dall'inizio posizioni eclettiche e ambivalenti, non ispirate a un credo ideologico ed espressione di un "populismo di centro" (Colloca e Corbetta 2015).

L'allargamento degli orientamenti postmaterialisti ha determinato l'affermazione tra i giovani di atteggiamenti favorevoli all'apertura, alla tolleranza, al cosmopolitismo ${ }^{1}$. Diversi fattori spingono in tale direzione: la crescita del livello di istruzione, l'essere socializzati all'idea di Europa, la curiosità verso la mobilità e la conoscenza delle lingue. Tuttavia, la prospettiva di una generazione di neo-elettori progressisti e post-ideologici contrasta (o, meglio, si combina) con la presenza di ampie componenti del mondo giovanile a basso titolo di studio ed economicamente fragili, che votano formazioni populiste, nativiste e anti-immigrazione (Miller-Idriss 2018; Uba e Bosi 2021). Nel caso dell'Italia, la penetrazione elettorale dei partiti di destra tra i giovani risale già agli anni novanta, con il forte sostegno raccolto da Alleanza Nazionale (Tuorto 2018). I successi più recenti della Lega e di Fratelli d'Italia segnalano, al tempo stesso, il

\footnotetext{
${ }^{1}$ Questo tratto è confermato dall'indagine Eurobarometro del 2019 (How do we build a stronger, more united Europe? The views of young people) su oltre 10.000 giovani di età compresa tra 15 e 30 anni. Si rimanda a: https://data.europa.eu/euodp/en/data/dataset/S2224_478_ ENG
}

consenso diffuso di orientamenti identitari e nazionalisti tra le nuove generazioni di elettori, ma anche una capacità di combinare posizioni diverse improntate, al tempo stesso, all'apertura e alla chiusura sui temi economici e culturali.

\section{STRUTTURA DEL LAVORO, IPOTESI E VARIABILI}

L'articolo si propone di ricostruire, con riferimento al contesto italiano contemporaneo, la relazione tra orientamenti di voto espressi dagli elettori e posizionamento sui temi politici.

Tre sono le ipotesi che muovono la nostra riflessione. La prima, di carattere più generale, sostiene che, coerentemente con quanto evidenziato dalla letteratura, gli elettori con preferenze di voto contrapposte tendano a distinguersi tra loro più sui temi culturali che su quelli economici. In particolare, i temi culturali maggiormente divisivi sono quelli che rimandano alla dimensione comunitaria-identitaria, e quindi all'immigrazione e all'integrazione europea.

La seconda ipotesi si riferisce alle differenze di età. In questo caso, assumiamo che siano soprattutto i giovani a manifestare incoerenza di posizione, in ragione del fatto che attingono a una cultura politica meno strutturata (sono entrati sulla scena in una fase di de-politicizzazione e de-ideologizzazione). Più che tra altri gruppi di elettori, ci attendiamo che tra i giovani l'indicazione della preferenza partitica sia scollegata rispetto al modo in cui interpretano le questioni che riguardano le libertà individuali, sociali, economiche.

Infine, come terza ipotesi, sosteniamo che a mostrare una struttura di posizioni meno congruente sui temi sia soprattutto l'elettorato giovanile dei partiti di centrodestra, che deve conciliare le dimensioni del cosmopolitismo, della tolleranza delle diversità e dei valori postmaterialisti presenti nella cultura di massa delle nuove generazioni con spinte tradizionaliste, anti-immigrazione e nativiste egualmente diffuse all'interno di quell'area politica.

La variabile dipendente utilizzata per l'analisi è la posizione degli elettori sui temi politici. Le preferenze sull'economia si riferiscono a un solo item, quello relativo alla classica alternativa tasse-servizi (ridurre le tasse anche al costo di ridurre i servizi pubblici vs estendere i servizi anche a costo di aumentare le tasse), ordinato secondo un range di posizioni che va da 1 a 7 (1 massimo pro-mercato e 7 massimo pro-Stato). Per quanto riguarda le altre dimensioni, abbiamo preso in esame le seguenti questioni: famiglie: tutela e difesa del modello tradizionale di famiglia vs. riconoscimento delle nuo- 
ve forme di unione familiare (scala 1-7); immigrazione: riceviamo troppi immigrati vs va bene come è adesso (scala 1-7); Europa: appartenenza alla UE è un bene vs. è un male ( 3 posizioni, con categoria intermedia "né un bene né un male"). Mentre la dimensione economica rimanda più chiaramente alla distinzione sinistra-destra, quella culturale può essere interpretata, seguendo la letteratura, in termini di contrapposizione tra autonomia individuale e fiducia nelle norme condivise collettivamente contrapposta a protezionismo, conservatorismo, populismo autoritario. Per semplificazione, applichiamo la stessa etichetta ideologica ai due assi e facciamo riferimento, anche sulla dimensione culturale, alla distinzione tra sinistra e destra, i cui significati si sono progressivamente estesi a temi diversi da quello economico originario (Van der Brug e Van Spanje 2009). Allo scopo di uniformare la metrica, le quattro scale sono state normalizzate (valori 0-10).

La variabile indipendente è la "preferenza di voto", ossia la propensione o disponibilità futura a votare $\mathrm{i}$ diversi partiti. La domanda rimanda a una scala di probabilità che va da 0 a 10 (10 esprime la certezza di votare un determinato partito e 0 la certezza di non votarlo). Tra le diverse probabilità presenti abbiamo preso in esame quelle relative ai principali partiti: Lega (LN), Fratelli d'Italia (FdI) e Forza Italia (FI) per il centrodestra, Partito Democratico (PD), Liberi e Uguali (LEU) e Movimento 5 stelle (M5S) per l'area opposta. A differenza della scelta di voto, l'utilizzo della PTV consente di considerare più propriamente tutte le opzioni che si prefigura l'elettorato riferite a una dimensione futura. Inoltre, permette di includere anche i partiti minori che difficilmente raggiungerebbero la numerosità campionaria di risposte con la domanda diretta sul partito votato. Ai fini dell'analisi ci limitiamo a considerare le singole propensioni, senza valutare in modo incrociato le disponibilità a scegliere più formazioni, e consideriamo come elettorato potenziale di un dato partito tutti i soggetti che indicano una disponibilità elevata di votarlo (da 8 a 10 sulla scala). ${ }^{2}$

Tra le altre variabili esaminate, l'età funziona da controllo. Le fasce considerate sono tre: 18-40, 41-60 e più di 60 anni. Il primo gruppo, dei più giovani, presenta una soglia superiore spostata in avanti rispetto ad altri studi. Questa decisione è derivata dalle esigenze di garantire un'elevata numerosità del sotto-campione e un

\footnotetext{
${ }^{2}$ Gli elettori potenziali identificati in questo modo (posizioni 8-10 della scala) tendono a segnalare un solo partito come scelta più probabile. La percentuale di chi indica analoga disponibilità a votare anche altro partito risulta sempre bassa (inferiore al 10\%) ad eccezione degli elettorati potenziali di Lega e Fratelli d'Italia che si sovrappongono in circa un quarto dei casi.
}

equilibrio numerico tra i gruppi di età prescelti. Si giustifica, inoltre, dalle particolari caratteristiche dei giovani italiani e dalle loro transizioni familiari posticipate che fissano il raggiungimento della piena età adulta ben oltre i $30 \mathrm{anni}^{3}$. Per quanto riguarda, infine, la fonte dati abbiamo utilizzato il dataset dell'indagine Itanes sull'opinione pubblica e il referendum costituzionale (rilevazione con tecnica CAWI). Le interviste sono state condotte nel settembre 2020 su un campione di partecipanti a un panel di intervistati nel 2016, 2018 e 2019. L'analisi si concentra esclusivamente sui dati del 2020; il numero di casi complessivo è compreso tra 1.600 e 1.700 .

\section{RISULTATI}

Il primo passaggio per comprendere come gli italiani si autorappresentano lo spazio politico è quello di rilevare le loro posizioni sulle issues e collegarle con gli orientamenti di voto. Il vincolo sempre più incerto tra elettori e partiti fa ritenere che questo legame si sia alterato, che le questioni chiave del dibattito pubblico vengano interpretate dagli elettori sulla base di valutazioni autonome, non collegate tra loro e non sempre guidate da un quadro politico-ideologico coerente riconducibile alla distinzione generale sinistra-destra.

I grafici riportati in fig.1 confermano parzialmente questa lettura. Due sono le tendenze che emergono. Innanzitutto, le posizioni assunte dagli elettorati potenziali dei vari partiti sull'economia risultano tutte racchiuse all'interno di un range piuttosto ristretto e concentrato attorno al centro della scala. Al crescere delle propensioni di voto per un dato partito gli orientamenti si spostano verso la polarità "più mercato e meno tasse" per chi sostiene i partiti di centro-destra e verso quella opposta favorevole all'intervento pubblico per gli elettori del centro-sinistra. Tuttavia, i due blocchi restano abbastanza vicini (non oltre le 2 posizioni di differenza sulla scala 0-10), distinti ma non contrapposti. Analogamente, l'elettorato del M5S esprime un posizionamento centrale tra liberismo e statalismo, senza mostrare alcuna evoluzione specifica (fig.1). Questa convergenza conferma, quindi, quanto già riscontrato a livello internaziona-

\footnotetext{
${ }^{3}$ Larticolo si concentra esclusivamente sulle differenze riconducibili al ciclo di vita (confronto tra fasce di età) e non prende in considerazione né l'effetto generazione né quello dovuto al periodo. Questa decisione deriva dalla natura dei dati utilizzati, che non consentono di ricostruire le risposte delle diverse coorti lungo un periodo elettorale ampio, né di considerare l'impatto degli eventi generali, di contesto, nelle varie fasi storiche. A prescindere da questi limiti dell'analisi, il disallineamento tra scelte di voto e posizione sui temi è un fenomeno relativamente recente (o comunque accentuatosi negli anni recenti) che rende in ogni caso incompleta un'analisi per generazione.
} 
le sulla scarsa tenuta del cleavage economico tradizionale, sia per i partiti mainstream sia per quelli sfidanti (Kitschelt 2004).

Per quanto riguarda i temi culturali la differenziazione è maggiore. Le posizioni su famiglia ed Europa risultano divisivi, mentre sull'immigrazione prevalgono orientamenti complessivamente contrari sebbene con sfumature all'interno del campione. Rispetto alle constituencies di Lega e FdI, il bacino di elettori potenziali di FI si distingue per una minore rilevanza delle posizioni pro-chiusura. Nell'altro campo la situazione è speculare: forte sostegno ai diritti per le nuove famiglie e per l'integrazione europea tra gli elettori del PD e di Leu, che contrasta con il posizionamento intermedio degli elettori del M5S (Fig. 1). Provando a sintetizzare questi primi risultati, non emergono elementi sufficienti a supporto dell'ipotesi di una disarticolazione complessiva dello spazio politico italiano, mentre appare confermata la prevalenza delle divisioni culturali su quelle economiche e anche l'esistenza di un'area di elettori, riconducibile al M5S e a FI, che appare più fluida nelle sue scelte.

Rispetto a questo quadro generale, come si collocano i giovani? Che tipo di profili politici esprimono? In che termini sono diversi da quelli del resto della popolazione? Per rispondere a questi interrogativi abbiamo calcolato i punteggi medi sulle issue degli elettori potenziali dei diversi partiti (quelli che hanno espresso una propensione a votarli superiore a 7 sulla scala $0-10$ ) distinte per fascia di età (Tab. 1). In generale, i giovani appaiono più orientati verso le polarità pro-Stato e pro libertarismo-apertura, con differenze contenute e che si colgono principalmente nel confronto con la fascia dei 41-60enni. L'età sembra contare assai poco per l'elettorato potenziale della Lega e di FdI, tra cui prevale una compattezza di posizioni in linea con l'orientamento ideologico dei due partiti. Unica eccezione riguarda il tema famiglie, su cui si riscontrano posizioni di apertura più accentuate tra $\mathrm{i}$ giovani. Ma su questo tema è soprattutto l'elettorato di Forza Italia a distinguersi, con punteggi nettamente più favorevoli tra la componente giovanile $(6,2)$ rispetto a quella adulta $(4,0)$ e agli over $60(3,7)$. Guardando all'area politica opposta, la dinamica cambia. Tra gli elettorati del PD e di Leu posizioni di apertura sui temi culturali e di sostegno allo Stato in economia riguardano in primis la fascia di età anziana, poi i giovani e decisamente meno i 41-60enni, la categoria che esprime la maggiore discontinuità con gli orientamenti tradizionali del centro-sinistra. Per quanto riguarda l'elettorato dei 5 stelle, infine, non emerge alcuna differenza riconducibile all'età. Nel complesso, anche l'ipotesi di una maggiore incoerenza del voto giovanile non trova pieno riscontro. Solo sul tema famiglie emergono posizioni significativa- mente diverse da quelle del resto dell'elettorato e tali da travalicare gli orientamenti di voto (in questo caso, del voto a destra).

L'analisi sin qui condotta ha interessato le singole tematiche, senza considerare l'articolazione complessiva delle posizioni. Abbiamo osservato in precedenza che un indicatore di cambiamento dello spazio politico è proprio la destrutturazione dell'allineamento sui temi. Il riferimento è, ad esempio, ad elettori che si collocano a destra sull'economia ma sono favorevoli all'immigrazione o, al contrario, che sono a sinistra sull'economia e sentono l'immigrazione come un problema. Un discorso analogo può essere sostenuto riguardo ai diritti delle nuove famiglie o e all'apertura-chiusura sull'Europa. Il passaggio ulteriore è quindi quello di costruire un indice sintetico che tenga conto di tutte le singole issues, classificate come coerenti o incoerenti sulla base della loro connotazione politica-ideologica. Per posizioni di sinistra sono stati considerati i punteggi 5-7 delle scale su economia, famiglie e immigrazione e la categoria di risposta "l'integrazione europea è un bene". La posizione di destra si riferisce ai punteggi 1-3 delle prime tre scale e alla categoria "l'integrazione europea è un male". Abbiamo infine trattato separatamente le categorie intermedie di ogni item (punteggio 4 delle prime tre scale e categoria di risposta "l'integrazione europea non è né un bene né un male") associandole a un profilo di centro o neutro. Il punteggio massimo dell'indice così costruito raggiunge il valore 4 nel caso di posizioni tutte allineate lungo la stessa direzione, fino a un punteggio minimo di 2 (massima differenza con solo due posizioni uguali). Nella tab.2 compare la distribuzione dei punteggi per classi di età senza alcun riferimento alla propensione di voto per i partiti. Lo scopo è infatti quello di capire se e come i temi si combinano tra loro e, solo in un secondo momento, di confrontare i diversi elettorati.

Un primo risultato da sottolineare riguarda l'incidenza di posizioni "congruenti". La quota di rispondenti le cui preferenze risultano tutte allineate lungo la stessa polarità è poco meno di un quinto del totale $(17,8 \%)$, con percentuali maggiori tra i giovani $(20,4 \%)$ rispetto alla fascia di età adulta $(15,1 \%)$. Si tratta, in questo caso, di un dato inatteso derivante da una forte differenziazione interna: a risultare allineate sono, infatti, le posizioni di sinistra (tutte e 4 le issue a sinistra: $14,5 \%$ ) e solo in minima parte quelle di destra $(4,6 \%)$. Questa caratteristica si ritrova anche nella categoria della "prevalenza" (3 posizioni su 4 nella stessa direzione). Complessivamente, i "congruenti" o "prevalenti" a sinistra corrispondono al $28 \%$ tra i $18-40$ enni e ad appena il $18 \%$ tra i $40-60$ enni che, al contrario, mostrano una percentuale più eleva- 

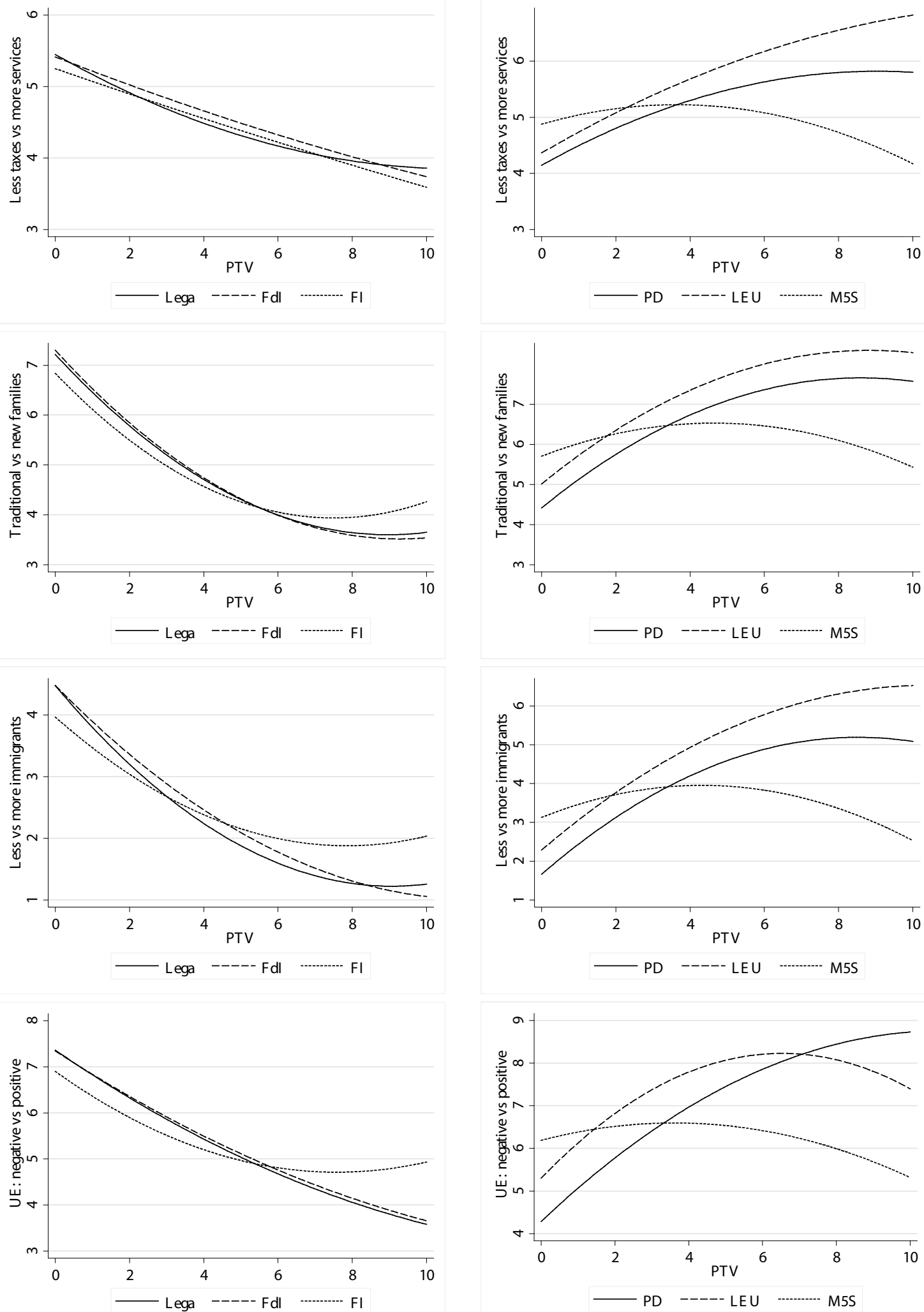

Figura 1. Posizione sui temi economici e culturali in base alla propensione di voto. Nota: Le scale di posizioni sui temi (asse Y) sono state trasformate da 1-7 a 0-10. La propensione di voto (asse X) va da 0 a 10. Fonte: Itanes 2020. 
Tabella 1. Punteggi medi di posizione sui temi per età e propensione di voto.

\begin{tabular}{lccccc}
\hline & \multicolumn{5}{c}{ Economia } \\
\cline { 2 - 6 } & $18-40$ & $41-60$ & Over 60 & Tot & $\begin{array}{c}\text { Test F } \\
\text { (sig.) }\end{array}$ \\
\hline Lega & 4,1 & 3,6 & 4,2 & 3,9 & $1,6(.198)$ \\
FdI & 4,6 & 3,3 & 4,3 & 3,9 & $5,2(.006)$ \\
FI & 4,4 & 3,7 & 3,1 & 3,8 & $0,4(.643)$ \\
PD & 6,2 & 5,4 & 6,0 & 5,8 & $2,1(.123)$ \\
Leu & 6,4 & 5,8 & 7,3 & 6,5 & $3,9(.025)$ \\
M5S & 4,9 & 4,2 & 4,0 & 4,3 & $1.3(.281)$ \\
\hline & & & & & \\
\hline & & & Famiglie & & \\
\cline { 2 - 6 } & & & & & Test F \\
& $18-40$ & $41-60$ & Over 60 & Tot & $($ sig. $)$ \\
\hline Lega & 4,3 & 3,4 & 3,6 & 3,6 & $0.9(.306)$ \\
FdI & 4,0 & 3,2 & 3,8 & 3.5 & $1,4(.257)$ \\
FI & 6,2 & 4,0 & 3,7 & 4,4 & $2,9(.056)$ \\
PD & 8,1 & 7,6 & 7,6 & 7,7 & $0,5(.577)$ \\
Leu & 7,9 & 7,4 & 8,7 & 8,1 & $1,6(.215)$ \\
M5S & 6,2 & 5,8 & 4,8 & 5,7 & $2,5(.086)$ \\
\hline
\end{tabular}

\begin{tabular}{lccccc}
\hline & \multicolumn{5}{c}{ Immigrazione } \\
\cline { 2 - 6 } & $18-40$ & $41-60$ & Over 60 & Tot & $\begin{array}{c}\text { Test F } \\
(\text { sig. })\end{array}$ \\
\hline Lega & 1,4 & 1,2 & 1,1 & 1,3 & $0,3(.716)$ \\
FdI & 1,4 & 1,1 & 1,0 & 1,2 & $0,4(.645)$ \\
FI & 2,7 & 1,6 & 1,7 & 1,9 & $1,2(.308)$ \\
PD & 5,6 & 4,5 & 5,6 & 5,3 & $4,8(.009)$ \\
Leu & 6,0 & 5,3 & 7,0 & 6,2 & $3,2(.047)$ \\
M5S & 2,5 & 2,8 & 2,8 & 2,7 & $0,1(.875)$ \\
\hline & & & & & \\
\hline & & & Europa & & \\
\cline { 2 - 6 } & & & & & Test F \\
& $18-40$ & $41-60$ & Over 60 & Tot & $($ sig. $)$ \\
\hline Lega & 3,4 & 3,7 & 4,0 & 3,7 & $0,4(.673)$ \\
FdI & 4,0 & 4,0 & 3,7 & 3,9 & $0.2(.835)$ \\
FI & 5,5 & 5,1 & 4,8 & 5,1 & $0,1(.852)$ \\
PD & 8,3 & 8,4 & 8,9 & 7,6 & $1,5(.218)$ \\
Leu & 7,5 & 7,3 & 8,7 & 8,0 & $1,7(.194)$ \\
M5S & 5,5 & 5,9 & 6,0 & 5,8 & $1.1(.873)$ \\
\hline
\end{tabular}

Nota: I punteggi sui temi sono calcolati su scale normalizzate (0-10). Per ogni partito vengono riportati i punteggi del sottocampione di elettori che hanno indicato un'elevata propensione di voto per il partito (posizioni da 8 a 10). Il test F si riferisce alla differenza tra le medie delle diverse classi di età per partito.

Fonte: Itanes 2020.

Tabella 2. Profili di posizione sui temi in base all'età.

\begin{tabular}{lcccc}
\hline & $18-40$ & $41-60$ & Over 60 & Tot \\
\hline Congruenza (4 su 4) & 20,4 & 15,1 & 19,1 & 17,8 \\
Posizioni di sinistra & 14,5 & 7,1 & 13,3 & 11,1 \\
Posizioni di destra & 4,6 & 6,5 & 5,0 & 5,5 \\
Posizioni di centro & 1,3 & 1,5 & 0,8 & 1,2 \\
Prevalenza (3 su 4) & 31,2 & 37,0 & 39,4 & 36,4 \\
Posizioni di sinistra & 13,8 & 11,5 & 15,5 & 13,5 \\
Posizioni di destra & 13,2 & 19,1 & 18,9 & 17,6 \\
Posizioni di centro & 4,2 & 6,4 & 5,0 & 5,3 \\
Incongruenza (2 su 4) & 48,4 & 47,9 & 41,5 & 45,8 \\
Tot & 100,0 & 100,0 & 100,0 & 100,0 \\
$\mathrm{~N}$ & 455 & 738 & 645 & 1838 \\
\hline
\end{tabular}

Nota: Per congruenti si intende 4 punteggi su 4 orientati nella stessa direzione (destra, sinistra o centro). Posizioni a destra: punteggi 1-3; posizioni a sinistra: punteggi 5-7; posizione di centro: punteggio 4. Prevalenti: 3 punteggi su 4 orientati nella stessa direzione. Incongruenti: al massimo 2 posizioni orientate nella stessa posizione. Temi: economia, famiglia, immigrazione, Europa. Fonte: Itanes 2020.

ta di allineati totalmente o parzialmente su posizioni di destra (26\%) (Tab. 2).
Le differenze tra giovani e adulti si possono cogliere anche confrontando le sequenze più ricorrenti di posizioni sui temi (dati non riportati in tabella). I giovani tendono a collocarsi più frequentemente a sinistra sull'economia e sulla famiglia e ad occupare posizioni di centro invece che di destra sul tema immigrazione. Altri profili diffusi sono quelli "a sinistra su famiglia ed Europa, al centro o a destra su economia e immigrazione" e "a sinistra sui temi culturali, al centro su economia". Al contrario, tra gli adulti prevalgono le combinazioni "destra su economia, famiglia, immigrazione, centro su Europa" e "sinistra su economia, famiglia, Europa, destra su immigrazione", o anche "destra su economia, immigrazione ed Europa, sinistra su famiglie". È evidente come sia soprattutto il tema immigrazione a spingere a destra anche quando le altre posizioni risultano di sinistra o al centro.

Come ultimo passaggio dell'analisi abbiamo collegato questi risultati agli orientamenti di voto. In fig.2 compaiono i valori predetti di congruenza sulle issues ottenuti attraverso regressioni lineari in cui la variabile dipendente è il punteggio stimato dall'indice (min-max congruenza, range normalizzato $0-1$ ), la variabile indipendente la propensione al voto (scala $0-10$ ), mentre la 

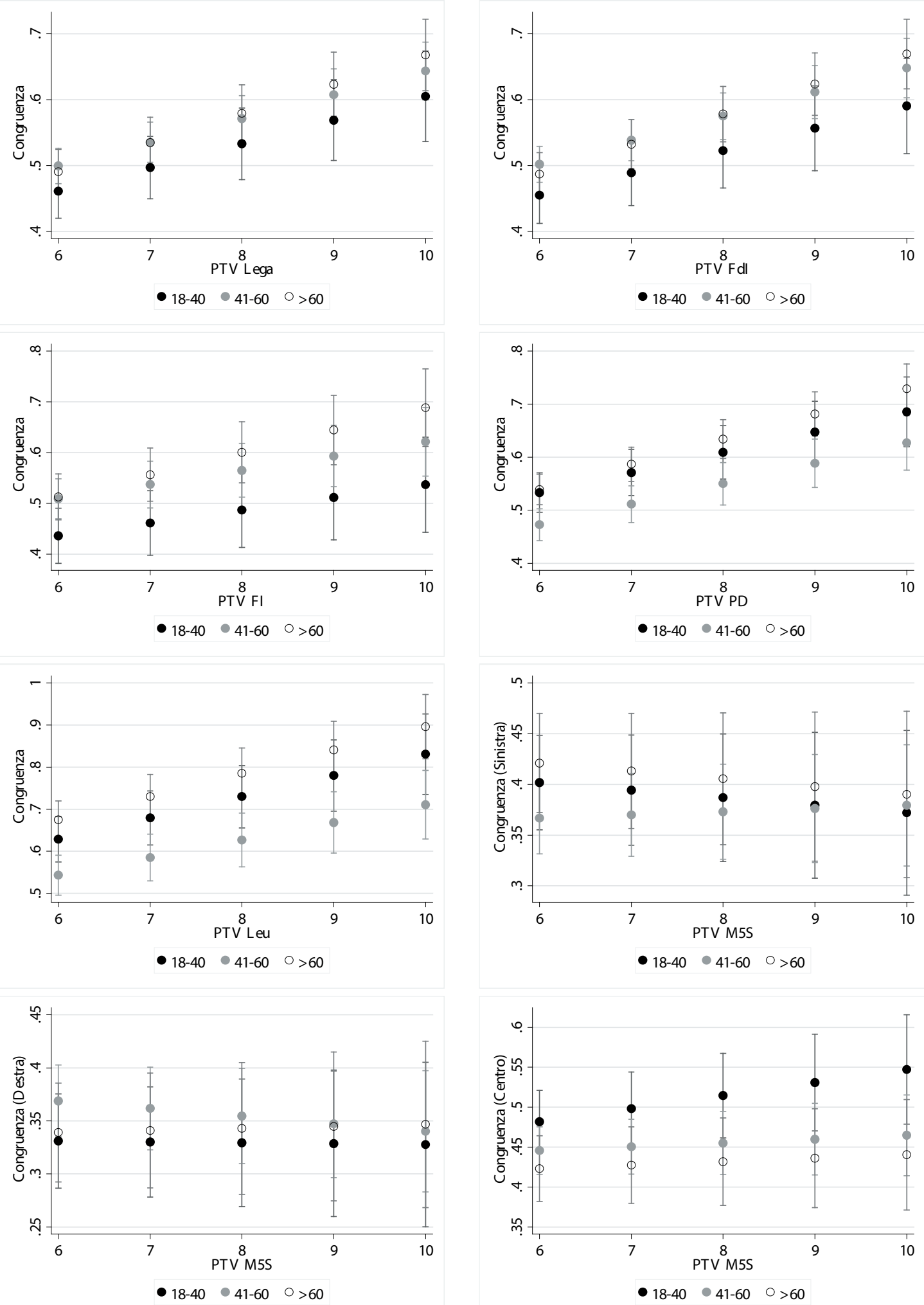

Figura 2. Congruenza sui temi in base alla propensione di voto e alla classe d’età. Punteggi predetti. Nota: Le figure riportano i punteggi di issue congruence stimati dalle regressioni lineari per le tre fasce di età al variare della propensione di voto (punteggi di PTV riportati: da 6 a 10). Fonte: Itanes 2020. 
classe di età è il fattore inserito nell'interazione (con le altre variabili sociodemografiche genere, zona di residenza, condizione occupazionale, titolo di studio presenti nei modelli come controllo). Abbiamo lanciato singole regressioni corrispondenti alle propensioni di voto per $\mathrm{i}$ diversi partiti: Lega, FdI, FI, PD, Leu, M5S. Mentre per i primi tre partiti l'indice di congruenza è stato calcolato sulla base delle posizioni di destra, per PD e Leu abbiamo considerato le posizioni di sinistra. Nel caso del M5S sono stata effettuate regressioni distinte con l'indice di coerenza a sinistra, a destra e al centro, in ragione della particolare collocazione ideologica dell'elettorato del partito. Le figure riportano l'andamento dei punteggi a partire dalla propensione di voto con punteggio 6 fino al massimo di 10.

Si può notare, innanzitutto, una tendenza che interessa elettorati politicamente contrapposti: al crescere della propensione di voto aumenta linearmente anche il grado di coerenza sui temi. La compattezza di posizioni è elevata tra gli elettorati del PD (punteggi da 0,5 a 0,7 su un massimo di 1 ) e di Leu (da 0,6 a 0,8 ), ma anche quelli di Lega e FdI raggiungono valori rilevanti $(0,5-$ 0,6 ) (Fig. 2). Un secondo risultato riguarda le differenze di punteggio in relazione all'età. Tra i rispondenti orientati verso i partiti del centro-destra, i giovani rappresentano sempre la categoria meno coerente. Nel caso di FI il divario è particolarmente ampio, sia perché gli anziani sono particolarmente allineati a destra, sia per la maggiore apertura dei giovani sui temi della famiglia e dell'integrazione europea. La stessa anomalia di posizioni non si riscontra tra gli elettorati del PD e Leu: i punteggi di coerenza sono elevati e seguono da vicino quelli dell'elettorato anziano. Non trova quindi conferma, all'interno di quest'area politica, la tesi del disallineamento dei giovani, mentre è nella fascia di età intermedia che si registra una maggiore discontinuità, dovuta soprattutto alle posizioni sull'immigrazione (alta incidenza di contrari) e sull'economia (molte posizioni centriste invece che stataliste).

Un discorso a parte va fatto, ancora una volta, per l'elettorato del Movimento 5 stelle che, assieme a quello di FI, mostra l'andamento più incerto. Se per FI la spiegazione è il gap di età (giovani ideologicamente fluidi, anziani compatti a destra), nel caso del M5S la questione è più complessa. I punteggi di congruenza sui temi risultano sempre bassi (circa la metà di quelli del resto del campione), sia considerando come riferimento l'allineamento a sinistra sia quello a destra. Tenendo conto delle caratteristiche del partito e dell'anomalo posizionamento sull'asse sinistra-destra già rilevato in altri studi (Colloca e Corbetta 2015), abbiamo riportato graficamente anche i punteggi di coerenza rispetto alle posizioni neutre, cor- rispondenti alle categorie centrali di ogni issue $e^{4}$. Mentre nel campione complessivo il peso di questi profili è irrilevante (cfr. Tab. 2), nel caso del M5S al crescere della propensione di voto per il partito aumenta il punteggio di congruenza "centrista" per i giovani (da 0.45 a 0.55 ). Questa relazione, anche se debole, indica un'attitudine specifica dell'elettorato pentastellato a collocarsi su posizioni non estreme rispetto ai temi politici analizzati. E il fatto che tale caratteristica interessi solo i giovani sta a segnalare una modalità peculiare di interpretare il cambiamento: non attraverso il disallineamento di posizioni tra le issue (alcune di sinistra, altre di destra), ma attraverso il non posizionamento.

\section{CONCLUSIONI}

L'articolo intendeva testare, con riferimento al quadro politico italiano del 2020, quanto le posizioni dei cittadini sulle questioni divisive del dibattito pubblico fossero organizzate in modo coerente e se questa coerenza si riflettesse anche in orientamenti di voto "corretti", ossia per quei partiti più vicini sul piano ideologico e in grado di rappresentare meglio le preferenze degli elettori nel loro complesso. Sullo sfondo di queste riflessioni c'è il dibattito, ampiamente sintetizzato nel paragrafo iniziale, sul declino dei cleavages tradizionali e l'emergere di nuove linee di divisione (quali appunto l'asse dell'apertura-chiusura), in grado di attraversare lo spazio politico tagliando le preferenze elettorali. Nell'articolo ci siamo posti, poi, la domanda su quanto le differenze di età abbiano contribuito ad alternare il legame tra elettori, temi e scelte partitiche.

L'analisi ha evidenziato la presenza di spazi politici distinti. Gli elettorati disponibili a votare partiti collocati in schieramenti opposti mostrano ancora posizioni diverse tra loro sulle principali questioni discusse nel dibattito pubblico. Per quanto riguarda l'età, il contributo fornito dai giovani al processo di destrutturazione dello spazio politico appare complessivamente ridimensionato. Il grado di coerenza sui temi è simile a quello delle altre classi di età. Emerge, inoltre, un'interessante differenza tra gli elettorati: mentre tra quelli vicini ai partiti di centro-sinistra i giovani mostrano una certa compattezza e una chiara collocazione lungo le polarità peculiari dell'area politica (su tutti gli item eccetto, parzialmente, per l'immigrazione), nel campo opposto (o quantomeno in una parte di questo campo e su alcune

\footnotetext{
${ }^{4}$ Le posizioni neutre sui temi corrispondono al punteggio 4 delle scale 1-7. Come nei casi precedenti, l'indice di coerenza di centro va da 0 a 1 , con 0 che corrisponde a nessuna risposta neutra sui temi e 1 a tutte le risposte neutre.
} 
dimensioni) il contributo della componente giovanile spinge verso l'allentamento dei confini.

Questa differenza tra elettorati e tra gruppi di età apre a una questione importante che riguarda non solo le preferenze individuali ma anche le strategie dei partiti. Un elettorato fedele, in grado di allineare correttamente le proprie posizioni e interpretare correttamente le posizioni delle varie forze politiche rappresenta una risorsa o un limite per partiti che tentano di allargare il proprio bacino tradizionale di consensi e/o crearne uno nuovo mai intercettato prima? Diversamente, un elettorato meno coerente risulta anche più volatile, stabilisce legami meno stabili con i partiti? Tali interrogativi si connettono evidentemente con la questione generazionale di cui ci siamo occupati nell'articolo. Mantenere una coerenza di posizioni può essere un fattore determinante per la scelta di voto (e anche per la decisione di andare a votare) se si guarda agli elettori anziani, socializzati nella politica dei grandi partiti, e probabilmente anche agli elettori di mezza età che hanno vissuto il bipolarismo della Seconda repubblica. Tra i giovani, invece, la situazione appare differente. È soprattutto nel campo del voto a destra che si pongono le questioni più interessanti. L'emergere di (alcune) posizioni moderatamente di apertura, in particolare rispetto ai temi delle famiglie e dell'Europa, sta a segnalare la presenza di una seppur minima complessità di dimensioni dentro il polo del cosiddetto autoritarismo culturale. Il rifiuto dei diritti delle minoranze espresso dalla spinta anti-immigrazione non risulta incompatibile con l'affermazione delle libertà personali, di cui l'estensione dei diritti alle nuove unioni familiari è una delle espressioni. Per i partiti dell'area di (centro) destra l'incoerenza giovanile su alcuni temi è il riflesso di una cultura progressista che tende a generalizzarsi e ad uscire dai suoi confini politico-partitici tradizionali. Al contrario, nel campo opposto è l'elettorato adulto a distanziarsi dal "seminato" coniugando orientamenti di segno contrastante tra loro e rispetto alla linea storicamente espressa dai partiti di riferimento. Resta un dato da confermare, con studi più approfonditi: il profilo della destra italiana, dopo la fase di successi negli anni novanta, non appare forse sufficientemente attraente per un vasto ambito di giovani a cui potrebbe non bastare la nettezza di posizioni sul tema immigrazione espresso dalle due formazioni più rappresentative, la Lega e Fratelli d'Italia.

A completamento di queste riflessioni è necessario richiamare nella discussione il caso del M5S. Di questo partito è nota la capacità di incorporare ampie fasce di voto giovanile (come è avvenuto clamorosamente nelle elezioni del 2013). Se è vero che i giovani ne rappresen- tano una constituency importante, i dati evidenziano anche come manchi ad essi un tratto ricorrente della politica giovanile, ossia il radicalismo. Al contrario, sono proprio i giovani del M5S a manifestare posizioni indistinte su tutti i temi, con la parziale eccezione dell'estensione dei diritti alle nuove famiglie. Questo tratto anomalo può rappresentare al tempo stesso un elemento di forza e di debolezza. Invece di alimentare la contrapposizione sui valori, come avviene tra i partiti sfidanti in tutta Europa, l'elettorato giovanile del M5S assume un profilo poco delineato e sempre coperto sul piano ideologico-valoriale, riproducendo una caratteristica già riscontrata da tempo rispetto al posizionamento sinistradestra. C'è da considerare, però, che l'analisi qui presentata non ha trattato la questione dell'antipolitica su cui potrebbe riversarsi il portato di radicalismo che non trova espressione sugli altri temi.

\section{BIBLIOGRAFIA}

Bartle, J., Sanders, D., Twyman, J. (2020), Authoritarian Populist Opinion in Europe, in Crewe. I., Sanders, D., Authoritarian Populism and Liberal Democracy, pp. 49-71.

Bornschier, S. (2010), The New Cultural Divide and the Two-Dimensional Political Space in Western Europe, in West European Politics 33,3, pp. 419-444.

Bornschier, S e Kriesi H. (2012), The populist right, the working class, and the changing face of class politics, in Rydgren J. (a cura di), Class Politics and the Radical Right. Abingdon and New York: Routledge, pp. 10-29.

Colloca, P. e Corbetta, P. (2015), Beyond protest: Issues and ideological inconsistencies in the voters of the Movimento 5 Stelle, in Tronconi, F., Beppe Grillo's Five Star Movement: Organisation, communication and ideology, Farnham, Ashgate, pp. 195-211.

Corbetta, P., Tuorto, D. e Cavazza, N. (2013), Parents and children in the political socialisation process: Changes in Italy over thirty-five years, in Abendschön, S., Growing into politics: Context and timing of political socialization, Ecpr Press, pp. 11-32.

Dalton, R. J. (1984), Cognitive mobilization and partisan dealignment in advanced industrial democracies, in The Journal of Politics, 46,1, pp. 264-284.

De Lange, S. L. (2007), A new winning formula? The programmatic appeal of the radical right, in Party Politics, 13, 4, pp. 411-435.

Dennison, J. and Geddes, A. (2019), A rising tide? The salience of immigration and the rise of anti-immigration political parties in Western Europe, The political quarterly, 90,1, pp. 107-116. 
De Sio, L. e Lachat, R. (2020), Issue competition in Western Europe: an introduction, in West European Politics, 43,3, pp. 509-517.

Downs, A. (1957), An Economic Theory of Democracy, New York, Harper and Row.

Franklin, M. N., Mackie, T.T. and Valen, H. (2009), Electoral change: Responses to evolving social and attitudinal structures in Western countries, Colchester, ECPR Press.

Franklin, M. (2004), Voter turnout and the dynamics of electoral competition in established democracies since 1945, Cambridge University Press.

Franklin, M. e Wessels, B. (2002), Learning Not to Vote: The Generational Basis of Turnout Decline in Established Democracies, paper presentato all'Annual meeting of the American Political Science Association.

Franklin, M. (1992), The decline of cleavage politics., in Franklin, M., Mackie, T. e Valen, H., Electoral Change: Responses to evolving social and attitudinal structures in Western countries, Cambridge, Cambridge University Press, pp. 383-405.

Fuchs, D. e Klingemann, H.D. (1990), The left-right sche$m a$, in Jennings, M.K., Continuities in political action: A longitudinal study of political orientations in three western democracies, Berlin-New York, Walter de Gruyter, pp. 203-234.

Garcia-Albacete, G. (2014), Young people's political participation in Western Europe: Continuity or generational change?, Basingstoke New York, Palgrave Macmillan.

Hooghe, L., Marks, G., e Wilson, C. J. (2002), Does Left/ Right Structure Party Positions on European Integration?, in Comparative Political Studies, 35, pp. 965-989.

Hooghe, L. e Marks, G. (2018), Cleavage theory meets Europe's crises: Lipset, Rokkan, and the transnational cleavage, in Journal of European Public Policy, 25, 1, pp. 109-135.

Inglehart, R., (1990), Values, ideology, and cognitive mobilization in new social movements., in Dalton, R. J. e Kuechler, M., Challenging the political order: new social and political movements in Western democracies, Oxford University Press, pp. 43-66.

Inglehart, R., Welzel C. (2005), Modernization, Cultural Change and Democracy: The Human Development Sequence. Cambridge: Cambridge university press.

Kitschelt, H (2004), Diversification and reconfiguration of party systems in postindustrial democracies, Bonn: Friedrich Ebert Stiftung.

Klingemann, H.D., Hofferbert, R.I., Budge, I. (1994), Parties, policies, and democracy, Westview Press.

Knutsen, O. (1995), Value orientations, political conflicts and left-right identification, in European Journal of Political Research, 28, pp. 63-93.
Kriesi, H., Grande, E., Lachat, R., Dolezal, M., Bornschier S. e Frey, T. (2006), Globalization and the Transformation of the National Political Space: Six European Countries Compared, in European Journal of Polical Research, 45, 6, pp. 921-956

Kriesi, H., Grande, E., Lachat, R., Dolezal, M., Bornschier S. e Frey, T. (2008). West European politics in the age of globalization, Cambridge: Cambridge University Press.

Kurella, A.-S., Rosset J. (2018), The rise of cultural issues as an opportunity for the Right? Insights from the 2015 Swiss election. Swiss Political Science Review, 24.4, 381-399.

Janmaat, J.G., Keating, A. (2019), Are today's youth more tolerant? Trends in tolerance among young people in Britain, Ethnicities 19.1, pp. 44-65.

Lau, R.R., Redlawsk, D.P. (1997), Voting correctly, in American Political Science Review, pp. 585-598.

Lau, R.R, Patel, P., Fahmy, D. F. e Kaufman, R. R (2014), Correct voting across thirty-three democracies: A preliminary analysis, in British Journal of Political Science, pp. 239-259.

Lefevere, J., Sevenans, J., Walgrave, S., Lesschaeve, C. (2019), Issue reframing by parties: The effect of issue salience and ownership, in Party Politics, 25, 4 pp. 507-519.

Louwerse, T., Andeweg, R.B. (2020), Measuring representation: policy congruence, Edward Elgar Publishing, 2020, in Cotta, M. e Russo, F. (a cura di), Research Handbook on Political Representation. Cheltenham, Edgar Elgar, pp. 276-288.

Maggini, N. (2016), Young People's Voting Behaviour in Europe: A Comparative Perspective, London, Palgrave McMillan.

Miller-Idriss, C. (2018), Youth and the radical right, in Rydgrin, J., The Oxford handbook of the radical right (2018), Oxford, Oxford University Press, pp. 348-365.

Miller, W.E, Shanks, J.M. e Shapiro, R.Y., (1996), The new American voter, London, Harvard University Press.

Mudde, C. (2007), Populist radical right parties in Europe, Cambridge, Cambridge University Press.

Pierce, R. (1999), Mass-elite issue linkages and the responsible party model of representation, in Miller, W., Pierce, R., Thomassen, J., Herrera, R. Holmberg, S., Esaisson, P. e Wessels, B., Policy representation in western democracies, Oxford, Oxford University Press, pp. 9-32.

Plutzer, E. (2002), Becoming a habitual voter: Inertia, resources, and growth in young adulthood, in American political science review, pp. 41-56.

Rabinowitz, G., Macdonald, S.E. (1989), A Directional Theory of Issue Voting, in American Political Science Review, 83, pp. 93-121 
Rubenson, D., Blais, A., Fournier, P., Gidengil, E., e Nevitte, N. (2004), Accounting for the age gap in turnout, in Acta Politica, 39, 4, pp. 407-421.

Schadee, H, Segatti, P., Vezzoni, C. (2019), Lapocalisse della democrazia italiana, Bologna, Il Mulino.

Sloam, J., Henn, M. (20019), Youthquake 2017: The rise of young cosmopolitans in Britain, Basingstoke, Springer Nature.

Tuorto, D. (2018), Lattimo fuggente: giovani e voto in Italia, tra continuità e cambiamento, Bologna. Il Mulino.

Uba, K. e Bosi, L. (2021), Explaining youth radicalism as a positioning of the self at opposite extremes, in Politics, https://doi.org/10.1177/0263395721990539.

Van der Brug, W. e Van Spanje, J. (2009), Immigration, Europe and the 'new'cultural dimension, in European Journal of Political Research, 48, 3, pp. 309-334.

Van der Brug, W. e Fennema, M. (2003), Protest or mainstream? How the European anti-immigrant parties have developed into two separate groups by 1999 , in European Journal of Political Research, 42,1, pp. 55-76.

Van Haute, E. (a cura di) (2016), Green parties in Europe, London, Routledge.

Walgrave, S., Lefevere, J. e Tresch, A. (2014), The limits of issue ownership dynamics: The constraining effect of party preference, in Journal of Elections, Public Opinion \& Parties, pp. 1-19.

Welzel, C., Inglehart, R.e Klingemann, H. D. (2003), Human development as a theory of social change: a cross-cultural perspective, in European Journal of Political Science, 42, 3, pp. 341-379.

Werts, H., Scheepers, P. e Lubbers, M. (2013), Euro-scepticism and Radical Right-Wing Voting in Europe, 2002-2008: Social Cleavages, Socio-Political Attitudes and Contextual Characteristics Determining Voting for the Radical Right, in European Union Politics, 14, 2, pp. 183-205. 


\section{APPENDICE}

Tabella 1. Modelli di regressione multipla. Variabile dipendente: issue congruency (a destra).

\begin{tabular}{|c|c|c|c|c|c|c|c|c|c|}
\hline & \multicolumn{3}{|c|}{ LN } & \multicolumn{3}{|c|}{ FdI } & \multicolumn{3}{|c|}{ FI } \\
\hline & Coef. B & St.err. & Sign. & Coef. B & St.err. & Sign. & Coef. B & St.err. & Sign. \\
\hline \multicolumn{10}{|l|}{ Eta: } \\
\hline $18-40$ & 0,000 & 0,000 & 0,000 & 0,000 & 0,000 & 0,000 & 0,000 & 0,000 & 0,000 \\
\hline $41-60$ & 0,038 & 0,021 & 0,074 & 0,031 & 0,022 & 0,157 & 0,055 & 0,023 & 0,015 \\
\hline$>60$ & $-0,020$ & 0,023 & 0,390 & $-0,038$ & 0,023 & 0,101 & $-0,036$ & 0,024 & 0,141 \\
\hline PTV LN (0-10): & 0,036 & 0,004 & 0,000 & 0,034 & 0,004 & 0,000 & 0,025 & 0,006 & 0,000 \\
\hline \multicolumn{10}{|l|}{ Eta*PTV: } \\
\hline $18-40$ & 0,000 & 0,000 & 0,000 & 0,000 & 0,000 & 0,000 & 0,000 & 0,000 & 0,000 \\
\hline $41-60$ & $-0,000$ & 0,005 & 0,997 & 0,003 & 0,005 & 0,615 & 0,003 & 0,007 & 0,667 \\
\hline$>60$ & 0,008 & 0,005 & 0,117 & 0,012 & 0,005 & 0,031 & 0,019 & 0,007 & 0,008 \\
\hline Genere & $-0,012$ & 0,014 & 0,364 & $-0,002$ & 0,014 & 0,873 & $-0,010$ & 0,015 & 0,510 \\
\hline Zona geopolitica & $-0,010$ & 0,005 & 0,768 & $-0,008$ & 0,003 & 0,567 & $-0,002$ & 0,000 & 0,866 \\
\hline Titolo di studio & $-0,011$ & 0,003 & 0,000 & $-0,013$ & 0,003 & 0,000 & $-0,017$ & 0,003 & 0,000 \\
\hline Condizione occupazionale & 0,006 & 0,016 & 0,707 & 0,006 & 0,016 & 0,720 & 0,010 & 0,017 & 0,553 \\
\hline Costante & 0,321 & 0,040 & 0,000 & 0,322 & 0,040 & 0,000 & 0,386 & 0,043 & 0,000 \\
\hline Adj R-squared & 0,226 & & & 0,224 & & & 0,117 & & \\
\hline $\mathrm{N}$ & 1636 & & & 1636 & & & 1636 & & \\
\hline
\end{tabular}

Tabella 2. Modelli di regressione multipla. Variabile dipendente: issue congruency (a sinistra).

\begin{tabular}{|c|c|c|c|c|c|c|}
\hline & \multicolumn{3}{|c|}{$\mathrm{PD}$} & \multicolumn{3}{|c|}{ Leu } \\
\hline & Coef. B & St.err. & Sign. & Coef. B & St.err. & Sign. \\
\hline \multicolumn{7}{|l|}{ Eta: } \\
\hline $18-40$ & 0,000 & 0,000 & 0,000 & 0,000 & 0,000 & 0,000 \\
\hline $41-60$ & $-0,063$ & 0,026 & 0,015 & $-0,033$ & 0,024 & 0,165 \\
\hline$>60$ & $-0,051$ & 0,029 & 0,083 & 0,017 & 0,026 & 0,517 \\
\hline PTV LN (0-10): & 0,038 & 0,005 & 0,000 & 0,051 & 0,006 & 0,000 \\
\hline \multicolumn{7}{|l|}{$\mathrm{Eta}^{\star P T V}:$} \\
\hline $18-40$ & 0,000 & 0,000 & 0,000 & 0,000 & 0,000 & 0,000 \\
\hline $41-60$ & 0,000 & 0,006 & 0,936 & $-0,009$ & 0,007 & 0,241 \\
\hline$>60$ & 0,009 & 0,006 & 0,097 & 0,005 & 0,007 & 0,510 \\
\hline Genere & $-0,013$ & 0,015 & 0,364 & 0,002 & 0,015 & 0,879 \\
\hline Zona geopolitica & 0,003 & 0,001 & 0,654 & $-0,009$ & 0,002 & 0,754 \\
\hline Titolo di studio & 0,019 & 0,003 & 0,000 & 0,019 & 0,003 & 0,000 \\
\hline Condizione occupazionale & $-0,005$ & 0,017 & 0,758 & $-0,012$ & 0,017 & 0,487 \\
\hline Costante & 0,219 & 0,043 & 0,000 & 0,230 & 0,043 & 0,000 \\
\hline Adj R-squared & 0,233 & & & 0,194 & & \\
\hline $\mathrm{N}$ & 1636 & & & 1636 & & \\
\hline
\end{tabular}


Tabella 3. Modelli di regressione multipla. Variabile dipendente: issue congruency (a sinistra, a destra, a centro).

\begin{tabular}{|c|c|c|c|c|c|c|c|c|c|}
\hline & \multicolumn{3}{|c|}{ M5s $(\sin )$} & \multicolumn{3}{|c|}{ M5s (des) } & \multicolumn{3}{|c|}{ M5s (cen) } \\
\hline & Coef. B & St.err. & Sign. & Coef. B & St.err. & Sign. & Coef. B & St.err. & Sign. \\
\hline \multicolumn{10}{|l|}{ Eta: } \\
\hline $18-40$ & 0,000 & 0,000 & 0,000 & 0,000 & 0,000 & 0,000 & 0,000 & 0,000 & 0,000 \\
\hline $41-60$ & $-0,099$ & 0,027 & 0,000 & 0,076 & 0,026 & 0,003 & 0,033 & 0,023 & 0,139 \\
\hline$>60$ & 0,021 & 0,028 & 0,457 & $-0,009$ & 0,027 & 0,750 & 0,013 & 0,024 & 0,576 \\
\hline PTV LN (0-10): & $-0,007$ & 0,005 & 0,155 & $-0,001$ & 0,005 & 0,864 & 0,016 & 0,004 & 0,000 \\
\hline \multicolumn{10}{|l|}{ Eta*PTV: } \\
\hline $18-40$ & 0,000 & 0,000 & 0,000 & 0,000 & 0,000 & 0,000 & 0,000 & 0,000 & 0,000 \\
\hline $41-60$ & 0,011 & 0,006 & 0,100 & $-0,006$ & 0,006 & 0,302 & $-0,012$ & 0,005 & 0,033 \\
\hline$>60$ & 0,000 & 0,007 & 0,965 & 0,003 & 0,007 & 0,683 & $-0,012$ & 0,006 & 0,045 \\
\hline Genere & $-0,001$ & 0,016 & 0,947 & $-0,018$ & 0,015 & 0,252 & 0,013 & 0,014 & 0,339 \\
\hline Zona geopolitica & $-0,010$ & 0,005 & 0,432 & $-0,006$ & 0,005 & 0,432 & $-0,01$ & 0,004 & 0,497 \\
\hline Titolo di studio & 0,024 & 0,003 & 0,000 & $-0,019$ & 0,003 & 0,000 & 0,000 & 0,003 & 0,927 \\
\hline Condizione occupazionale & $-0,022$ & 0,019 & 0,246 & 0,007 & 0,018 & 0,690 & $-0,006$ & 0,016 & 0,881 \\
\hline Costante & 0,337 & 0,047 & 0,000 & 0,463 & 0,045 & 0,000 & 0,370 & 0,040 & 0,000 \\
\hline Adj R-squared & 0,046 & & & 0,028 & & & 0,033 & & \\
\hline $\mathrm{N}$ & 1636 & & & 1636 & & & 1636 & & \\
\hline
\end{tabular}

